\title{
Certification Trails and Software Design for Testability
}

\author{
Gregory F. Sullivan ${ }^{1}$ \\ Dept. of Computer Science \\ Johns Hopkins University \\ Baltimore, MD 21218
}

\author{
Dwight S. Wilson ${ }^{2}$ \\ Dept. of Computer Science \\ Johns Hopkins University \\ Baltimore, MD 21218
}

Gerald M. Masson ${ }^{3}$

Dept. of Computer Science

Johns Hopkins University

Baltimore, MD 21218

\begin{abstract}
This paper investigates design techniques which may be applied to make program testing easier. We present methods for modifying a program to generate additional data which we refer to as a certification trail. This additional data is designed to allow the program output to be checked more quickly and effectively. Certification trails $[14,16]$ have heretofore been described primarily from a theoretical perspective. In this paper, we report on a comprehensive attempt to assess experimentally the performance and overall value of the certification trail method. The method has been applied to nine fundamental, well-known algorithms for the following problems: convex hull, sorting, huffman tree, shortest path, closest pair, line segment intersection, longest increasing subsequence, skyline, and voronoi diagram. Run-time performance data for each of these problems is given, and selected problems are described in more detail. Our results indicate that there are many cases in which certification trails allow for significantly faster overall program execution time than a 2-version programming approach, and also give further evidence of the breadth of applicability of this method.

Keywords: Software design for testability, software fault detection, certification trails, error monitoring, design diversity, data structures.
\end{abstract}

\section{Introduction}

We have examined a wide variety of fundamental algorithms to determine how they can be redesigned to allow for easier testability. To make the problem of testing the correctness of the output of a program more tractable we have found it is desirable to modify the program so that it generates additional data which we refer to as a certification trail. This additional data is designed to allow the program output to be checked

\footnotetext{
${ }^{1}$ Research partially supported by NSF Grants CCR-8910569 and CCR-8908092 and an IBM Teclunology Interchange Program Graut.

2 Research partially supported by NSF Grant CCR-8910569 and an IBM Technology Interchange Program Grant.

${ }^{3}$ Research partially supported by NASA Grant NSG 1442 and an IBM Technology Interchange Program Grant.
}

more quickly and effectively. Our previous work on certification trails emphasized a theoretical perspective in which we proved that the asymptotic time complexity of the testing process could be reduced $[14,16]$. In this paper, we report on implementations of the certification trail method so as to assess experimentally with run-time data the performance and overall value of the technique. We have implemented the certification trail method for nine fundamental and well-known algorithms of broad importance and applicability. For each algorithm, we have produced three implementations: a version which produces the output; a version which produces the output and generates a certification trail; and a version which checks the output while utilizing the certification trail. Specifically, algorithms for the following problems are analyzed: huffman tree, shortest path, sorting, closest pair, line segment intersection, convex hull, longest increasing subsequence, skyline, and voronoi diagram. The scope of the algorithms considered gives credibility to the overall applicability of the certification trail method. Furthermore, comparisons of run-time data for each of the three versions of each of the algorithms considered reveal many cases in which an approach using certification trails allows for significantly faster overall program execution time than a 2-version programming approach.

\section{Introduction to Certification Trails}

First, let us consider a basic method which is used to perform testing to detect software faults called $\mathrm{N}$ version programming $[1,2]$. This method utilizes $N$ teams of programmers, each independently implementing separate programs based on a problem specification. The programs are executed on the same input and the outputs are compared. Errors caused by software faults are detected whenever the independently written programs do not generate coincident errors. Thus the technique exploits design diversity. Also, note that the method can detect hardware faults which affect the separate executions in distinct ways causing distinct outputs. It is particularly valuable for detecting errors caused by transient fault phenomena. The $\mathrm{N}$-version programming method can be used to detect faults af 
discuss a general technique for construction of certification trails for algorithms using a wide range of data structures. This technique is used to implement the certification trails for several of our examples.

We provide a full description of the algorithm for the convex bull problem which generates a certification trail and a full description of the algorithm which uses that trail. Because of space considerations the discussion of the other algorithms is abbreviated. In some cases references to previous publications or technical reports which describe the algorithms more fully are given.

The algorithms we have chosen to implement are not always the algorithms which have the smallest asymptotic time complexity. Often the asymptotically fastest algorithms have large constants of proportionality which make them slower on the data sizes we examined. We modifed and used some programs from major software distributions such as quicker-sort from a Berkeley Unix distribution. Fortune's algorithm for computing the Voronoi diagram was obtained from an Internet site at AT\&T Bell Labs. Other algorithms were based on textbook discussions. It should be stressed bere that this research is continuing as we further increase our corpus of algorithm and datastructure implementations.

\subsection{Explanation of timing data}

We have collected timing data for the algorithms on a Sun SPARCstation ELC with 16MB of RAM. The system was run as a standalone machine in single user mode during the timing experiments. Timing data was obtained through the getrusage() system call. The user times are reported in the data.

Much of the data presented in the timing table is essentially self-explanatory relative to the certification trail technique and algorithms considered. However, a brief discussion of the table entries is appropriate.

The column labelled Basic contains timing data which gives the execution time of the algorithm in producing the output without the generation of the certification trail. All timing data is listed in seconds.

The Primary Execution (Prim. Exec.) column gives the execution time of the algorithm in producing the output with the additional overhead of generating the certification trail.

The Secondary Execution (Sec. Exec.) column gives the execution time of the algorithm in producing the output while using the certification trail.

The Percent Savings ( $\%$ Sav.) column records the percentage of the execution time savings which is gained by using the certification trail method as compared to 2-version programming approach. This as- sumes that both versions take approximately the same amount of time to execute.

The Speedup column is the ratio of the run times of the Basic Algorithm and the Secondary Execution.

For the Huffman tree data, the input size for the Huffman tree program is the number of nodes. Each node is given a frequency, chosen uniformly from the integers $\{1,2, \ldots, n\}$. $n$ was also selected to be the number of nodes.

For the shortest path table, there are two numbers associated with the input size, the first is the number of vertices in the graph, the second the number of edges. A graph with the required edges is selected uniformly from the set of all such graphs, then tested for connectedness in order to assure that paths exist to all vertices.

For the geometric algorithms, the input size is the number of points (or lines) in the original data set. Point set input was generated by choosing points with integer coordinates uniformly over a large square (typically $1,000,000$ by $1,000,000$ or larger square). For the Line Segment Intersection problem, lines were generated by picking a line segment start point uniformly from a large square and picking offsets for $x$ and $y$ coordinates from a smaller range to give the end point of the line segment. This was done to bound the line length and avoid data sets resulting in a quadratic number of intersections.

Data for the longest increasing subsequence problem was produced by generating a random permutation of $[1 . . N]$ for input size $N$.

Sorting was performed on an array of pointers to structures. It was assumed that each structure contains an extra integer field for use in generating the certification trail. Sorting was performed on integer keys, though the technique can be used with a more complex key (in fact, using complex keys is very likely to increase the speedup achieved). Integers were chosen uniformly from interval $[1.1,000,000,000]$.

\subsection{Convex Hull Example}

The convex hull problem is fundamental in the field of computational geometry. Our certification trail solution is based on a convex hull algorithm due to (iraham [6] called Graham's Scan. For basic definitions in computational geometry see the text of Preparata and Shamos[11]. For simplicity in the discussion which follows we will assume the points are in general position, e.g., no three points are collinear. It is not hard to remove this restriction.

Definition 4.1 The convex hull of a set of points, $T$, in the Euclidean plane is defined as the smallest convex polygon enclosing all the points. This polygon is unique 
fod its vertices are a subset of the points in $T$. It is pecified by a counterclockwise sequence of its vertices.

The algorithm given below constructs the convex bull incrementally in a counterclockwise fashion. The fust step of the algorithm selects an "extreme" point and calls it $p_{1}$. The next two steps sort the remaining points. The order of the points is determined by the dopes of the line segments formed by joining each point to $p_{1}$. It is not hard to show that after these three steps the points when taken in order, $p_{1}, p_{2}, \ldots, p_{n}$, form a imple polygon; although this polygon may not be convex. The Graham Scan algorithm traverses this polycon, removing points until the resulting polygon is convex. The main FOR loop iteration adds vertices to the polygon under construction and the inner WHILE loop removes vertices from the construction. A point is removed when the angle test performed at line 6 reveals What the angle at that vertex is obtuse. It is easy to demonstrate that when a point is removed, it must fall within the triangle defined by three other points, $p_{1}$ and the two points that were adjacent to the point removed. When the main FOR loop is complete the convex hull Has been constructed. The execution of this algorithm W demonstrated in Figure 2. For each removed point, - the associated triangle is indicated in bold lines, and in the text below the diagram. Our certification trail relies the fact that that these triangles can be determined quickly.

$3:$

Algorithm CONVEXHULL $(T)$

Input: Set of points, $T$, in $R^{2}$

Output: Counterclockwise sequence of points in

$R^{2}$ which define the convex hull of $T$

Let $p_{1}$ be the point with the largest

$x$ coordinate (and smallest $y$ to break ties)

For each point $p$ (except $p_{1}$ ) calculate

the slope of the line through $p_{1}$ and $p$

1. Sort the points (except $p_{1}$ ) from smallest

1." slope to largest. Call them $p_{2}, \ldots, p_{n}$

$q_{1}:=p_{1} ; q_{2}:=p_{2} ; q_{3}:=p_{3} ; m=3$

FOR $k=4$ to $n$ DO

1. WHILE the angle formed by

$q_{m-1}, q_{m}, p_{k}$ is $\geq 180$ degrees

$\therefore$ DO $m:=m-1$ END

7. $m:=m+1$

$8 q_{m}:=p_{k}$

END FOR

10 FOR $i=1$ to $m$ DO, OUTPUT $\left(q_{i}\right)$

END FOR

END CONVEXHULL

First execution: In this execution the code CONVEXHULL is used. The certification trial is generated
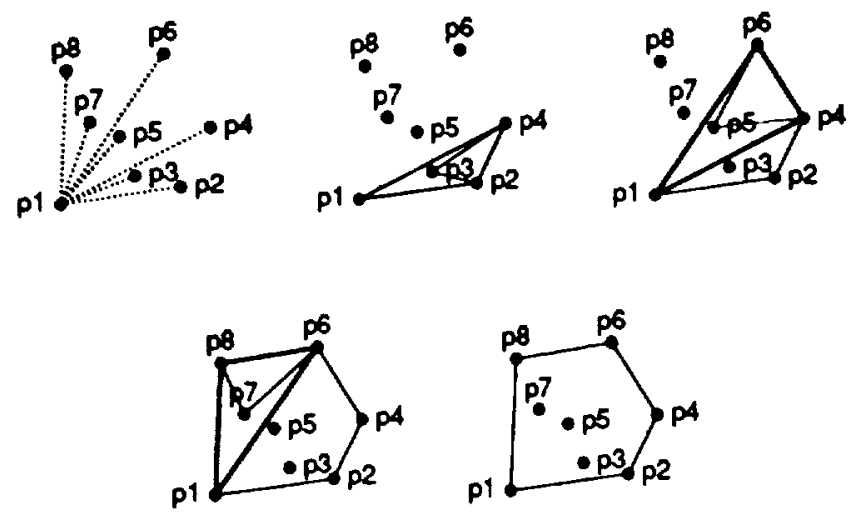

Figure 2: Convex hull example.

Point not on

Three surrounding points

convex hull

$\begin{array}{ll}p_{3} & p_{1}, p_{2}, p_{4} \\ p_{5} & p_{1}, p_{4}, p_{6} \\ p_{7} & p_{1}, p_{6}, p_{8}\end{array}$

by adding an output statement within the WHILE loop. Specifically, if an angle of less than 180 degrees is found in the WHILE loop test then the four tuple consisting of $q_{m}, q_{m-1}, p_{1}, p_{k}$ is output to the certification trail. The final convex hull points $q_{1}, \ldots, q_{m}$ are also output to the certification trail. Strictly speaking the trail output does not consist of the actual points in $R^{2}$. Instead, it consists of indices to the original input data. This means if the original data consists of $p_{1}, p_{2}, \ldots, p_{n}$ then rather than output the element in $R^{2}$ corresponding to $p_{i}$ the number $i$ is output.

Second execution: Let the certification trail consist of a set of four tuples, $\left(x_{1}, a_{1}, b_{1}, c_{1}\right),\left(x_{2}, a_{2}, b_{2}, c_{2}\right)$, $\ldots,\left(x_{r}, a_{r}, b_{r}, c_{r}\right)$ followed by the supposed convex hull, $q_{1}, q_{2}, \ldots, q_{m}$. The code for CONVEXBULL is not used in this execution. Indeed, the algorithm is dramatically different than CONVEXHULL.

It consists of five checks on the trail data.

- First, it checks that there is a one to one correspondence between the input points and the points in $\left\{x_{1}, \ldots, x_{r}\right\} \cup\left\{q_{1}, \ldots, q_{m}\right\}$.

- Second, it checks that for each $i \in\{1, \ldots, r\}, a_{i}$, $b_{i}$, and $c_{i}$ are among the input points.

- Third, the algorithm checks that for eacb $i \in$ $\{1, \ldots, r\}, x_{i}$ lies within the triangle defined by $a_{i}, b_{i}$, and $c_{i}$. 
- Fourth, the algorithm checks that for each triple of counterclockwise consecutive points on the supposed convex hull, the angle formed by the points is less than or equal to 180 degrees.

- Fifth, it checks that there is a unique point among the points on the supposed convex hull which is a local maxima. We say a point $q$ on the hull is a local maxima if its predecessor in the counterclockwise ordering has a strictly smaller $y$ coordinate and its successor in the ordering has a smaller or equal $y$ coordinate.

If any of these checks fail then execution halts and "error" is output. Otherwise the convex hull read from the trail is output. As mentioned above, the trail data actually consists of indices into the input data. This does not unduly complicate the checks above; instead it makes them easier. The correctness and adequacy of these checks must be proven. A complete formal proof is beyond the scope of this paper, instead a brief outline of the proof will be given.

Using our formal definition of certification trails, let $\mathbf{D}$ be the set of all finite planar point sets $T$. Let $\mathbf{S}$ be the set of convex polygons, with vertices in counterclockwise order (the restriction to counterclockwise ordering makes the convex hull unique). Then the problem we are considering is $B U L L: \mathbf{D} \rightarrow \mathrm{S}$ where $\operatorname{HULL}(T)$ is the polygon in $\mathbf{S}$ that forms the convex hull of $T$.

The description of the algorithms above defines functions $F_{1}$ and $F_{2}$. We must show that both conditions of Definition 3.2 hold. The following two lemmas, which we state without proof, are required.

Lemma 4.2 Let $P$ be a polygon on $n$ points $p_{1}, p_{2}, \ldots, p_{n} . P$ is a convex polygon iff $P$ is simple and each angle $p_{i} p_{j} p_{k}$ is less than or equal to 180 degrees, where $i$ is in $1,2, \ldots n, j=(i+1) \bmod n$, and $k=(i+2) \bmod n$.

Lemma 4.3 If $P$ is a non-simple polygon, then either $P$ has more than one local maxima, or the interior angle at some veriex is greater than 180 degrees.

These are deceptively simple statements. Though they are intuitively obvious, a formal proof is difficult. It is interesting to note that some computer graphics texts give an incorrect test for determing convexity of a polygon by omitting the check for simplicity required by Lemma 4.2 .

Recall that the first condition is:

For all $d \in \mathrm{D}$ there exists $s \in \mathrm{S}$ and $t \in \mathbf{T}$ such that $F_{1}(d)=(s, t)$ and $F_{2}(d, t)=s$ and $(d, s) \in \mathbf{P}$.
Intuitively, this means that if both executions perform correctly then they will both output the convex hull of the input, which is unique. Note that generation of the certification trail does not affect the output of the Graham Scan algorithm. Thus the condition on $F_{1}(d)$ is satisfied by the correctness of the (iraham Scan algorithm, the proof of which is well known [11]. To show that $F_{2}(d, t)=s$, note that a copy of $s$ is contained on the trail $t$. Our description of $F_{2}(d, t)$ states that $s$ is output unless one of the five checks above fails. It is trivial to verify that the first three of these checks must be satisfied. The fourth check cannot fail, since the polygon described by $s$ is convex (because $(d, s) \in \mathbf{P})$. Similarly, if the fifth check fails, then the polygon described by $s$ has two local maxima, and this is not possible for a convex polygon.

The second condition is:

For all $d \in \mathbf{D}$ all $t \in \mathbf{T}$ either $\left(F_{2}(d, t)=s\right.$ and $(d, s) \in \mathbf{P})$ or $F_{2}(d, t)=$ error.

Intuitively, this means that given an input and arbitrary trail, $F_{2}(d, t)$ produces a solution to the problem or flags an error.

Our definition of $F_{2}(d, t)$ states that the polygon $Q$ stored on the trail is output unless one of the five checks fails. We must therefore demonstrate that if all five checks succeed, then $Q$ is the convex hull of the input points $d$. Let $H$ be the convex hull of the points $d$. The first condition guarantees that every point in $d$ is classified as a hull point or an interior point. The second condition guarantees that the triangles used to identify interior points are formed from input points, and the third check verifies that the interior points are indeed inside their respective triangles. Note that we do not attempt to verify that the triangles used are the ones that would be produced by $F_{1}(d)$. In general, for a given interior point, there may be several triangles of input points in which it is contained. Together, the first three conditions imply that all points in $H$ are also in $Q$, since it is impossible for a hull point to be contained in a triangle. Note that these three checks do not exclude the possibility that interior points are present in $Q$, nor do they guarantee that the ordering of the hull points in $Q$ is correct. The final two checks will accomplish this. If the last two checks are satisfied, Lemma 4.3 states that $Q$ is simple, and therefore it must be convex by Lemma 4.2 .

Thus, $Q$ is a convex polygon whose vertex set is a superset of the vertices of $H$, i.e., $H$ is contained in $T$. This implies that no other point from the input set may be a vertex of $Q$, since any input point that is not a hull point is interior to $H$ and therefore interior to $Q$. Finally, it is clear that the ordering of the vertices of $Q$ and $H$ must be the same (although there 
- might appear to be two possible orderings, clockwise and counterclockwise, a clockwise ordering will fail the fourth check). Therefore if all five checks succeed, then - the output of $F_{2}(d, t)$ will be the convex hull of $d$.

This demonstrates that the algorithms described meet the conditions of Definition 3.2, and are therefore certification trail solution to the convex hull problem.

Time complexity: In the first execution the sorting of the input points takes $O(n \log (n))$ time where $n$ is the number of in put points. One can show that this cost -dominates and the overall complexity is $O(n \log (n))$.

It is possible to implement the second execution so bat all five checks are done in $O(n)$ time. The first two hecks may be done in linear time since the certification trail contains indices into the input data. The third .nd fourth checks require a constant time calculation at ach point. Finally, the uniqueness of the local maxima is clearly checkable in linear time.

Order-of-Magnitude Testing Speedup: It hould be noted that for the convex hull problem, we wre seeing an order of magnitude speedup for reasonable sized problems. We believe this offers a dramatic emonstration of the efficiency of our proposed software -sting technique using certification trails in comparison with the 2-version programming technique.

\begin{tabular}{|c|c|c|c|c|c|}
\hline Size & Basic & $\begin{array}{c}\text { Prim. Exec. } \\
\text { (Also Gen. } \\
\text { Trail) }\end{array}$ & $\begin{array}{c}\text { Sec. } \\
\text { Exec. }\end{array}$ & $\begin{array}{c}\% \\
\text { Sav. }\end{array}$ & Speedup \\
\hline 5000 & 0.64 & 0.67 & 0.08 & 41.41 & 8.00 \\
\hline 10000 & 1.38 & 1.40 & 0.17 & 43.12 & 8.12 \\
\hline $\mathbf{2 5 0 0 0}$ & $\mathbf{3 . 8 9}$ & 3.84 & 0.46 & 44.73 & 8.46 \\
\hline 50000 & $\mathbf{8 . 4 4}$ & 8.50 & 0.85 & 44.61 & 9.93 \\
\hline 00000 & 17.36 & 17.68 & 1.65 & 44.33 & 10.52 \\
\hline
\end{tabular}

Table 1: Convex Hull

\subsection{Sorting Example}

This important problem has a massive literature. In trrs section we will discuss how to apply the certification trail approach to the sorting problem. Let us ume that the sorting algorithm takes as input an ar$r-$ of $n$ elements and outputs an array of $n$ elements. The algorithm is supposed to place the data in nond-reasing order.

To design a certification trail algorithm we must discover the nature of the data that should be included in the certification trail to allow quick computation of he final output sorted array. Suppose that we deci. to use the output array itself as the certification trail. We note that it is easy to check that this array is in on-decreasing order by simply performing a single pass over the array. Unfortunately, it is considerably more difficult to make sure that this array contains exactly the same elements as the original input array. Indeed, this problem has a lower bound time complexity of $\Omega(n \log (n))$ in a comparison based model.

Because of this difficulty we use the permutation of the elements defined by the input and output data arrays as the certification trail. This permutation is computed by attaching an Item Number field to the data elements before sorting. The $i$-th item receives item number $i$. After the elements are sorted, the permutation from input to output is obtained by reading the Item Numbers from the elements in their new order.

The second execution reads the permutation from the trail and verifies that it is a permutation on $\mathrm{n}$ elements, i.e., that no numbers are repeated or omitted. This permutation is used to rearrange the input elements in linear time. Finally the algorithm checks that these elements are now in non-decreasing order.

\begin{tabular}{|c|c|c|c|c|c|}
\hline Size & Basic & $\begin{array}{c}\text { Prim. Exec. } \\
\text { (Also Gen. } \\
\text { Trail) }\end{array}$ & $\begin{array}{c}\text { Sec. } \\
\text { Exec. }\end{array}$ & $\begin{array}{c}\% \\
\text { Sav. }\end{array}$ & Speedup \\
\hline 10000 & 0.28 & 0.30 & 0.04 & 39.29 & 7.00 \\
\hline 50000 & 1.80 & 1.90 & 0.19 & 41.94 & 9.47 \\
\hline 100000 & 3.96 & 4.08 & 0.41 & 43.31 & 9.66 \\
\hline 500000 & 23.95 & 24.69 & 2.14 & 43.99 & 11.19 \\
\hline 1000000 & 50.23 & 51.57 & 4.38 & 44.31 & 11.47 \\
\hline
\end{tabular}

Table 2: Sort

\subsection{Certification Trails For Abstract Data Types}

Before we present the rest of our example algorithms we discuss a general technique applicable to many algorithms and data structures.

An abstract data type is a data object or set of data objects together with a group of operations for manipulating the object(s). Each operation takes a (possibly empty) set of arguments, and some, but not necessarily all, operations return answers. Many algorithms make extensive use of abstract data types.

We describe a method for automatically generating a certification trail for an algorithm which uses an abstract data type. This is done by modifying the abstract data type operations, so that during the first execution they generate a certification trail, and during the second execution they use the certification trail. Otherwise, these operations are identical to the original abstract data type operations, i.e., they take the same type of arguments and have the same return types. The object of creating and using the certification trail is to 
allow a more efficient implementation of the abstract data type during the second execution.

We illustrate this technique for the following abstract data type which we call Ordered Collection. An Ordered Collection will contain a set of pairs $(i, x)$ where $i$ is an item number, and $x$ is a real number value. (This selection is made for simplicity of description, the elements being stored could be more complex). No two elements of the set may have the same item number, though several items may have a common value. We define a total ordering on pairs by $(i, x)<\left(i^{\prime}, x^{\prime}\right)$ iff $x<x^{\prime}$ or $x=x^{\prime}$ and $i<i^{\prime}$

The following operations are defined on an Ordered Collection:

INSERT $(i, x)$ Add the element $(i, x)$ to the set.

DELETE(i) Delete the element with item number $i$ from the set.

PREDECESSOR(i) Let $(i, x)$ be the element in the set with item number $i$. This operation returns its predecessor, that is, the largest pair less than $(i, x)$. A special value SMALLEST is returned if $(i, x)$ is the smallest element in the set.

MIN Return the smallest element in set.

NEAREST $(x)$ Return the element from the set with value closest to $x$. If there is a tie, return the element with the smallest item number.

This small set of operations is being chosen for concreteness, several additional operations could be easily defined. If an error occurs during any of these operations, for example, inserting pairs with duplicate item numbers or attempting to delete a non-existent item, then the program terminates indicating an error.

These operations may be modified to produce a certification trail during the first execution by modifying the INSERT $(i, x)$ and NEAREST $(x)$ operations to do the following (in addition to their normal function):

INSERT $(i, x)$ After adding this element to the set, perform a PREDECESSOR(i) operation and write the item number of the answer to the certification trail.

NEAREST $(x)$ Write the item number of the answer to the certification trail.

A typical implementation of an abstract data type supporting the above operations would require $\Omega(n \log (n))$ time to process a sequence of $n$ operations. By using the certification trail, we can achieve linear time for $n$ operations during the second execution. This includes the time necessary to check the trail for correctness as well as use it.

The implementation of the Ordered Collection for the second execution will be a structure called an indexed linked list. This is a doubly linked list, along with an array Items of pointers, indexed by item number. The $i$-th element in this array points to the list node for the element with item number $i$ (or is NULL if no element in the list has item number $i$ ). This allows us to find an element in constant time given its item number. The elements themselves are maintained in ascending order (according to the pair ordering given above) on a doubly linked list, i.e., each element has pointers to its successor and predecessor. In addition to the array, we maintain a variable Start, which stores the item number of the first element in the list.

The abstract data type operations for the second execution are defined as follows:

$\operatorname{INSERT}(i, x)$ Read the item number $p$ from the trail. $p$ is the item number that would be the predecessor of $(i, x)$ if it were in the set. Items[p] points to the list node for the element with index $p$, call this element $\left(p, x_{p}\right)$. We can insert $(i, x)$ after this node using ordinary list operations. Before doing so, however, we make three checks:

i. Check that Items[i] is currently NULL, i.e., there is not currently an element with item number $i$ in the set.

ii. Check that $(i, x)$ is greater than $\left(p, x_{p}\right)$.

iii. (heck that $(i, x)$ is less than the successor of $\left(p, x_{p}\right)$

If these checks are satisfied, then $(i, x)$ may be inserted after $\left(p, x_{p}\right)$. Set Items $[i]$ pointing to the list node for $(i, x)$.

Note that special cases occur at the beginning and end of the list. We omit the specifics of these cases, mentioning only that Start must be updated for insertions at the front of the list.

DELETE(i) Check that Items[i] is not NULL, i.e., there is an element with item number $i$ currently in the set. If so, remove it from the linked list, and set Items[i] to NULL. If we remove the first element of the list we must also update Start.

PREDECESSOR(i) Items[i] points to the element with item number $i$, and its predecessor may be found by following the appropriate pointer.

MIN The variable Start indicates the item number of the first element on the list, i.e., the minimum element. Items[Start] therefore points to this element. 

Huffman tree and which yield excellent data compression ratios. The tree structure and the code design are based on the frequencies of individual characters in the data to be compressed. Here we are concerned exclusively with the Huffman tree. See [7] for information about the coding application.

Definition 4.4 The Huffman tree problem is the following: Given a sequence of frequencies (positive integers) $f[1], f[2], \ldots, f[n]$, construct a tree with $n$ leaves and with one frequency value assigned to each leaf so that the weighted path length is minimized. Specifically, the tree should minimize the following sum: $\sum_{l, \in L \text { LAF }} \operatorname{len}(i) f[i]$ where LEAF is the set of leaves, len(i) is the length of the path from the root of the tree to the leaf $l_{i}, f[i]$ is the frequency assigned to the leaf $l_{i}$.

A full description of the method we employ to generate and use a certification trail is detailed in a technical report [15].

\begin{tabular}{|c|c|c|c|c|c|}
\hline Size & Basic & $\begin{array}{c}\text { Prim. Exec. } \\
\text { (Also Gen. } \\
\text { Trail) }\end{array}$ & $\begin{array}{c}\text { Sec. } \\
\text { Exec. }\end{array}$ & $\begin{array}{c}\text { \% } \\
\text { Sav. }\end{array}$ & Speedup \\
\hline 5000 & 0.81 & 0.87 & 0.16 & 36.42 & 5.06 \\
\hline 10000 & 1.76 & 1.86 & 0.33 & 37.78 & 5.33 \\
\hline 25000 & 6.01 & 6.30 & 1.02 & 39.10 & 5.89 \\
\hline 50000 & 10.62 & 11.14 & 1.70 & 39.55 & 6.25 \\
\hline
\end{tabular}

Table 4: Huffman tree

\subsection{Other problems}

We report timing data for five other problems, the "Manhattan skyline" problem, computation of Voronoi diagrams, longest increasing subsequence, the closest pair problem, and line segment intersection. Space permits only a brief description of these problems, rather than a full exposition of the certification trail techniques used.

The "Manhattan skyline" problem is: Given a set of rectangles with collinear bottom edges, compute the polygonal outline of the union of the rectangles [9].

The Voronoi diagram is a fundamental concept in computational geometry [11]. Given a set of points $P$ in the plane, the Voronoi diagram is a partition of the plane into regions such that each region consists of all points closer to a given $p \in P$ than to any other other point in $P$. Computation of the Voronoi diagram is an important step in many problems involving point location.

The next problem we consider is, given a sequence of integers, find the longest (not necessarily unique) strictly increasing subsequence.

\begin{tabular}{|c|c|c|c|c|c|}
\hline Size & Basic & $\begin{array}{c}\text { Prim. Exec. } \\
\text { (Also Gell. } \\
\text { Trail) }\end{array}$ & $\begin{array}{c}\text { Sec. } \\
\text { Exec. }\end{array}$ & $\begin{array}{c}\% \\
\text { Sav. }\end{array}$ & Speedup \\
\hline 1000 & 0.27 & 0.26 & 0.12 & 29.63 & 2.25 \\
\hline 5000 & 1.69 & 1.65 & 0.57 & 34.32 & 2.96 \\
\hline 10000 & 3.91 & 3.72 & 1.14 & 37.85 & 3.43 \\
\hline 15000 & 6.08 & 5.78 & 1.77 & 37.91 & 3.44 \\
\hline 20000 & 8.53 & 8.27 & 2.33 & 37.87 & 3.66 \\
\hline
\end{tabular}

Table 5: Skyline

\begin{tabular}{|c|c|c|c|c|c|}
\hline Size & Basic & $\begin{array}{c}\text { Prim. Exec. } \\
\text { (Also Gen. } \\
\text { Trail) }\end{array}$ & $\begin{array}{c}\text { Sec. } \\
\text { Exec. }\end{array}$ & $\begin{array}{c}\% \\
\text { Sav. }\end{array}$ & Speedup \\
\hline 100 & 0.04 & 0.04 & 0.03 & 12.50 & 1.33 \\
\hline 500 & 0.24 & 0.26 & 0.19 & 6.25 & 1.26 \\
\hline 1000 & 0.51 & 0.51 & 0.39 & 11.76 & 1.31 \\
\hline 5000 & 2.75 & 2.82 & 2.03 & 11.82 & 1.35 \\
\hline 10000 & 5.79 & 5.89 & 4.06 & 14.08 & 1.43 \\
\hline 50000 & 40.15 & 40.63 & 22.00 & 22.00 & 1.83 \\
\hline
\end{tabular}

Table 6: Voronoi Diagram

\begin{tabular}{|c|c|c|c|c|c|}
\hline Size & Basic & $\begin{array}{c}\text { Prim. Exec. } \\
\text { (Also Geln. } \\
\text { Trail) }\end{array}$ & $\begin{array}{c}\text { Sec. } \\
\text { Exec. }\end{array}$ & $\begin{array}{c}\% \\
\text { Sav. }\end{array}$ & Speedup \\
\hline 10000 & 0.13 & 0.14 & 0.04 & 30.77 & 3.25 \\
\hline 50000 & 0.78 & 0.81 & 0.22 & 33.97 & 3.55 \\
\hline 100000 & 1.61 & 1.70 & 0.44 & 33.54 & 3.66 \\
\hline 500000 & 9.17 & 9.32 & 2.22 & 37.08 & 4.13 \\
\hline 1000000 & 18.66 & 19.58 & 4.46 & 35.58 & 4.18 \\
\hline
\end{tabular}

Table 7: Longest Increasing Subsequence

Given a set of points $P$ in the plane, the Closest Pair problem is that of finding the pair of points with minimum distance over all pairs in the set.

\begin{tabular}{|c|c|c|c|c|c|}
\hline Size & Basic & $\begin{array}{c}\text { Prim. Exec. } \\
\text { (Also Gen. } \\
\text { Trail) }\end{array}$ & $\begin{array}{c}\text { Sec. } \\
\text { Exec. }\end{array}$ & $\begin{array}{c}\% \\
\text { Sav. }\end{array}$ & Speedup \\
\hline 10000 & 0.26 & 0.27 & 0.07 & 34.62 & 3.71 \\
\hline 50000 & 1.45 & 1.55 & 0.36 & 34.14 & 4.03 \\
\hline 100000 & 3.06 & 3.26 & 0.72 & 34.97 & 4.25 \\
\hline 500000 & 16.84 & 18.02 & 3.62 & 35.75 & 4.65 \\
\hline
\end{tabular}

Table 8: Closest Pair

Given a set of line segments in the plane, the line intersection problem is the problem of determining all intersections of line segments in this set.

For the first four problems, algorithms running in $O(n \log (n))$ time were implemented for the first execution. The second execution, using certification trails, runs in linear time. The first execution algorithm used for line intersection runs in $(O((k+n) \log (n))$ time where $k$ is the number of intersections and $n$ the number of points. The second execution runs in $O(k+n)$ time. Note that $k$ may be quadratic in $n$. 


$=$\begin{tabular}{|c|c|c|c|c|c|}
\hline Size & Basic & $\begin{array}{c}\text { Prim. Exec. } \\
\text { (Also Gen. } \\
\text { Trail) }\end{array}$ & $\begin{array}{c}\text { Sec. } \\
\text { Exec. }\end{array}$ & $\begin{array}{c}\% \\
\text { Sav. }\end{array}$ & Speedup \\
\hline 1000 & 0.47 & 0.49 & 0.04 & 43.62 & 11.75 \\
\hline 2500 & 1.45 & 1.53 & 0.12 & 43.10 & 12.08 \\
\hline 5000 & 3.33 & 3.47 & 0.26 & 43.99 & 12.81 \\
\hline 10000 & 7.72 & 7.88 & 0.60 & 45.08 & 12.87 \\
\hline 25000 & 24.00 & 24.12 & 1.75 & 46.10 & 13.71 \\
\hline
\end{tabular}

Table 9: Line Segment Intersection

\section{Concluding Discussion}

Certification trails have heretofore been discussed principally from a theoretical perspective. In this pa- per we have presented experimental timing data which illustrates the advantages of the certification trail technique for software testing over the 2-version programming technique. We have further presented techniques and analytical results for several new algorithms which further support the significance of the certification trail lechnique by demonstrating its broadening applicability. It should be appreciated that the scope of our experimental investigation is not limited to the algoithms considered here; numerous other algorithms we Iave considered could have been discussed, and we continue to work on new applications. It should also be nointed out that in addition to the timing experiments eported here, software fault injection experiments have also been conducted which verify the detection capabilities of the certification trail method. The breadth of pplicability of the certification trail technique contines to expand along with the credibility of its advantages. Increasingly, the certification trail method can he viewed as a competitive software testing alternative.

\section{References}

"1] Avizienis, A., "The N-version approach to fault tolerant software," IEEE Trans. on Software Engineering, vol. 11, pp. 1491-1501, Dec., 1985.

2] Chen, L., and Avizienis A., "N-version programming: a fault tolerant approach to reliability of software operation," 1978 Fault Tol. Comp. Symp., pp. 3-9, IEEE

Computer Society Press, 1978.

[3]

Dijkstra, E. W., "A note on two problems in connexion with graphs," Numer. Math. 1, pp. 269-271, 1959.

.

Fortune, S. "A Sweepline Algorithm for Voronoi Diagrams," Algorithmica, pp. 153-174, 2, 1987.

- J Fredman, M. L., and Willard, D. E., "Transdichotomons algorithms for minimum spanning trees and shortest paths," Proc. S1st IEEE Foundations of Computer Science, pp. 719-725,1990.
[6] Graham, R. L., "An efficient algorithm for determining the convex hull of a planar set", Information Process ing Letters, pp. 132-133, 1, 1972.

[7] Huftman, D., "A method for the construction of minimum redundancy codes", Proc. IRE, pp 1098-1101, 40, 1952 .

[8] Johnson, B., Design and analysis of fault tolerant digital systems Addison-Wesley, Reading, MA, 1989.

[9] Manber U., Introduction to Algorithms AddisonWesley, Reading, MA, 1989.

[10] Nievergelt, J., and Hinrichs, K. H., Algorithns and Data Structures With Applications to Graphics and Geometry, Prentice Hall, NJ 1993

[11] Preparata F. P., and Shamos M. I., Computational geometry, Springer-Verlag, New York, NY, 1985.

[12] Sedgewick, R., "Implementing quicksort programs," Comm. of the $A C M$, pp. 847-857, 21(10), 1978.

[13] Siewiorek, D., and Swarz, R., The theory and practice of reliable design, Digital Press, Bedford, MA, 1982.

[14] Sullivan, G.F., and Masson, G.M., "Using certification trails to achieve software fault tolerance," Digest of the 1990 Fault Tolerant Conputing Symposium, pp. 423431, IEEE Computer Society Press, 1990.

[15] Sullivan, G.F., and Masson, G.M., "Using certification trails to achieve software fault tolerance," Department of Computer Science Technical Report JHU $89 / 26$, Johns Hopkins University, Baltimore, Maryland, 1989.

[16] Sullivan, G.F., and Masson, G.M., "Certification trails for data structures," Digest of the 1991 Fault Tolerant Computing Symposium, pp. 240-247, IEEE Computer Society Press, 1991.

[17] Tarjan, R. E., Data Structures and Network Algorithms, Society for Industrial and Applied Mathematics, Philadelphia, PA, 1983. 